Kragujevac Journal of Mathematics

Volume 46(1) (2022), Pages 149-165.

\title{
SOME RESULTS ON POST-WIDDER OPERATORS PRESERVING TEST FUNCTION $x^{r}$
}

\author{
VIJAY GUPTA ${ }^{1}$ AND GANCHO TACHEV ${ }^{2}$
}

\begin{abstract}
In the present paper, we consider Post-Widder operators and its modified form which preserve the test function $x^{r}, r \in \mathbb{N}$. We estimate direct results in terms of modulus of continuity for the modified operators. Also, some estimates for polynomially bounded functions and linear combinations are considered. Our estimates improve in some sense the previous results for the original Post-Widder operators.
\end{abstract}

\section{INTRODUCTION}

The Post-Widder operators for $n \in \mathbb{N}$ and $x>0$ considered by Widder [18] is defined by

$$
P_{n}(f, x)=\frac{1}{n !}\left(\frac{n}{x}\right)^{n+1} \int_{0}^{\infty} t^{n} e^{-\frac{n t}{x}} f(t) d t .
$$

These operators preserve constant functions only. The $q$ analogue of these operators was recently studied by Aydin et al. [3]. Earlier Rathore and Singh [15] (also for related results see [9]) established an asymptotic formula, and deduced inverse and saturation theorems in simultaneous approximation. They considered a parameter $p$ and defined the operators in following way

$$
P_{n}^{p}(f, x)=\frac{1}{(n+p) !}\left(\frac{n}{x}\right)^{n+p+1} \int_{0}^{\infty} t^{n+p} e^{-\frac{n t}{x}} f(t) d t .
$$

Key words and phrases. Post-Widder operators, exponential functions, moments, polynomially bounded functions, linear combinations.

2010 Mathematics Subject Classification. Primary: 41A25. Secondary: 41A36.

DOI 10.46793/KgJMat2201.149G

Received: May 15, 2019.

Accepted: September 19, 2019. 
The special case $p=0$ provides the operator (1.1) and for $p=-1$, these operators reduce to the operators due to May [13], which preserve the linear functions and considered earlier in the book of Ditizian and Totik [5], by Li and Wang in [12], also in the papers of Draganov and Ivanov [6,7]. Rempulska and Skorupka in [16] further modified the Post-Widder operators of the form considered by May [13] in order to preserve the test function $e_{2}$, where $e_{r}(x)=x^{r}$. It was observed in [16] that the modified form provide better approximation results over the form of [13], but in that case the modified form loses the preservation of test function $e_{1}$. It may be observed that at a time only two preservations can be made either constant and $e_{1}$ or constant and the function $e_{r}, r>1, r \in \mathbb{N}$. Here we deal with the modification of Post-Widder operators which preserve constants and $e_{r}, r \in \mathbb{N}$.

Following [16], the $r$-th order moment $\mu_{r}^{P_{n}}(x)=P_{n}\left(e_{r}, x\right)$, where $e_{r}(t)=t^{r}, r \in$ $\mathbb{N} \cup\{0\}$ are given by

$$
P_{n}\left(e_{r}, x\right)=\frac{1}{n !}\left(\frac{n}{x}\right)^{n+1} \int_{0}^{\infty} t^{n} e^{-\frac{n t}{x}} t^{r} d t=\frac{1}{n !}\left(\frac{n}{x}\right)^{n+1} \int_{0}^{\infty} t^{n+r} e^{-\frac{n t}{x}} d t .
$$

Put $n t / x=u$ implying $(n / x) d t=d u$, thus

$$
\begin{aligned}
P_{n}\left(e_{r}, x\right) & =\frac{1}{n !}\left(\frac{n}{x}\right)^{n+1} \int_{0}^{\infty}\left(\frac{x}{n}\right)^{n+r+1} u^{n+r} e^{-u} d u \\
& =\frac{1}{n !}\left(\frac{x}{n}\right)^{r} \Gamma(n+r+1) \\
& =\frac{1}{n !}\left(\frac{x}{n}\right)^{r}(n+r) ! \\
& =\frac{(n+r)(n+r-1) \cdots(n+1) n !}{n !} \cdot \frac{x^{r}}{n^{r}}=\frac{(n+1)_{r} x^{r}}{n^{r}},
\end{aligned}
$$

where $(n)_{r}=n(n+1)(n+2) \cdots(n+r-1)$.

$$
\mu_{r}^{P_{n}}(x)=\frac{(n+1)_{r} x^{r}}{n^{r}}
$$

with $(n)_{0}=1$. If the central moments are denoted by $T_{m}^{P_{n}}(x)=P_{n}\left((t-x)^{m}, x\right)$, then

$$
\begin{aligned}
& T_{1}^{P_{n}}(x)=\frac{x}{n}, \\
& T_{2}^{P_{n}}(x)=\frac{(n+2) x^{2}}{n^{2}} .
\end{aligned}
$$

From the results given in [5, Ch. 9], denoting the space of all real valued continuous and bounded functions on $(0, \infty)$ by $C_{B}(0, \infty)$ for every $f \in C_{B}(0, \infty)$ and $\delta>0$ there holds

$$
|f(t)-f(x)| \leq \omega(f, \delta)\left(\frac{|t-x|}{\delta}+1\right)
$$


where $\omega(f, \cdot)$ is the first order modulus of continuity of $f$ defined by

$$
\omega(f, \delta)=\sup _{0<h \leq \delta, x \geq 0}|f(x+h)-f(x)| .
$$

Thus,

$$
\begin{aligned}
\left|P_{n}(f, x)-f(x)\right| & \leq \frac{1}{n !}\left(\frac{n}{x}\right)^{n+1} \int_{0}^{\infty} t^{n} e^{-\frac{n t}{x}}|f(t)-f(x)| d t \\
& \leq \frac{1}{n !}\left(\frac{n}{x}\right)^{n+1} \int_{0}^{\infty} t^{n} e^{-\frac{n t}{x}} \omega(f, \delta)\left(\frac{|t-x|}{\delta}+1\right) d t \\
& \leq \omega(f, \delta)\left(\frac{\sqrt{T_{2}^{P_{n}}(x)}}{\delta}+1\right) .
\end{aligned}
$$

Choosing $\delta=\sqrt{T_{2}^{P_{n}}(x)}$, we immediately get

$$
\left|P_{n}(f, x)-f(x)\right| \leq 2 \omega\left(f, \frac{\sqrt{n+2}}{n} x\right) .
$$

\section{Modified Post-Widder Operators Preserving $e_{r}$}

Let us consider that the Post-Widder operators preserve the test function $x^{r}, r \in \mathbb{N}$, then we start with the following form

$$
\widetilde{P}_{n, r}(f, x)=\frac{1}{n !}\left(\frac{n}{a_{n, r}(x)}\right)^{n+1} \int_{0}^{\infty} t^{n} e^{-\frac{n t}{a_{n}, r(x)}} f(t) d t .
$$

Here $\widetilde{P}_{n, r}$ preserves constants for any positive function $a_{n, r}(x)$. Then

$$
\begin{aligned}
x^{r}=\widetilde{P}_{n, r}\left(e_{r}, x\right) & =\frac{1}{n !}\left(\frac{n}{a_{n, r}(x)}\right)^{n+1} \int_{0}^{\infty} t^{n+r} e^{-\frac{n t}{a_{n, r}(x)}} d t \\
& =\frac{(n+r) !}{n !}\left(\frac{a_{n, r}(x)}{n}\right)^{r}=(n+1)_{r}\left(\frac{a_{n, r}(x)}{n}\right)^{r},
\end{aligned}
$$

implying

$$
a_{n, r}(x)=\frac{n x}{\left((n+1)_{r}\right)^{1 / r}} .
$$

Thus, our modified operators $\widetilde{P}_{n, r}, r \in \mathbb{N}, x \in(0, \infty)$, take the following form

$$
\widetilde{P}_{n, r}(f, x)=\int_{0}^{\infty} k_{n}(x, t) f(t) d t
$$

where

$$
k_{n}(x, t)=\frac{1}{n !}\left[\frac{\left((n+1)_{r}\right)^{1 / r}}{x}\right]^{n+1} t^{n} e^{-\frac{t}{x}\left((n+1)_{r}\right)^{1 / r}},
$$


with $\widetilde{P}_{n, r}(f, 0)=f(0)$ which preserve the function $x^{r}$ and the constant function.

Following (1.2), the $m$-th order moments are given by

$$
\widetilde{P}_{n, r}\left(e_{m}, x\right)=\frac{(n+1)_{m}\left(a_{n, r}(x)\right)^{m}}{n^{m}}=\frac{(n+1)_{m}}{\left((n+1)_{r}\right)^{m / r}} x^{m} .
$$

Lemma 2.1. The first few images of monomials are given by

$$
\begin{aligned}
\widetilde{P}_{n, r}\left(e_{0}, x\right) & =1, \\
\widetilde{P}_{n, r}\left(e_{1}, x\right) & =\frac{(n+1)}{\left((n+1)_{r}\right)^{1 / r}} x, \\
\widetilde{P}_{n, r}\left(e_{2}, x\right) & =\frac{(n+1)(n+2)}{\left((n+1)_{r}\right)^{2 / r}} x^{2} .
\end{aligned}
$$

Remark 2.1. We may point out here that when $r=1$, then the operator $\widetilde{P}_{n, 1}$ preserves constant as well as linear functions. When $r=2$ these preserve constant and test function $x^{2}$.

Lemma 2.2. If we denote the central moment as $T_{m}^{\widetilde{P}_{n, r}}(x)=\widetilde{P}_{n, r}\left((t-x)^{m}, x\right)$, then we have the following recurrence relation:

$T_{m+1}^{\widetilde{P}_{n, r}}(x)=\frac{x^{2}}{\left((n+1)_{r}\right)^{1 / r}}\left[T_{m}^{\widetilde{P}_{n, r}}(x)\right]^{\prime}+\left[\frac{(n+1)}{\left((n+1)_{r}\right)^{1 / r}}-1\right] x T_{m}^{\widetilde{P}_{n, r}}(x)+\frac{m x^{2} T_{m-1}^{\widetilde{P}_{n, r}}(x)}{\left((n+1)_{r}\right)^{1 / r}}$.

In particular

$$
\begin{aligned}
T_{1}^{\widetilde{P}_{n, r}}(x)= & {\left[\frac{(n+1)}{\left((n+1)_{r}\right)^{1 / r}}-1\right] x, } \\
T_{2}^{\widetilde{P}_{n, r}}(x)= & {\left[\frac{(n+1)_{2}}{\left((n+1)_{r}\right)^{2 / r}}-2 \frac{(n+1)}{\left((n+1)_{r}\right)^{1 / r}}+1\right] x^{2}, } \\
T_{4}^{\widetilde{P}_{n, r}}(x)= & {\left[\frac{(n+1)_{4}}{\left((n+1)_{r}\right)^{4 / r}}-4 \frac{(n+1)_{3}}{\left((n+1)_{r}\right)^{3 / r}}+6 \frac{(n+1)_{2}}{\left((n+1)_{r}\right)^{2 / r}}-4 \frac{(n+1)}{\left((n+1)_{r}\right)^{1 / r}}+1\right] x^{4}, } \\
T_{6}^{\widetilde{P}_{n, r}}(x)= & {\left[\frac{(n+1)_{6}}{\left((n+1)_{r}\right)^{6 / r}}-6 \frac{(n+1)_{5}}{\left((n+1)_{r}\right)^{5 / r}}+15 \frac{(n+1)_{4}}{\left((n+1)_{r}\right)^{4 / r}}\right.} \\
& \left.-20 \frac{(n+1)_{3}}{\left((n+1)_{r}\right)^{3 / r}}+15 \frac{(n+1)_{2}}{\left((n+1)_{r}\right)^{2 / r}}-6 \frac{(n+1)}{\left((n+1)_{r}\right)^{1 / r}}+1\right] x^{6} .
\end{aligned}
$$

For any $r \in \mathbb{N}$ we have $T_{m}^{\widetilde{P}_{n, r}}(x)=O\left(n^{-[(m+1) / 2]}\right)$.

Proof. The kernel $k_{n}(x, t)$ of our modified operators $\widetilde{P}_{n, r}$, satisfy the following identity

$$
x^{2} \frac{\partial}{\partial x} k_{n}(x, t)=\left[\left(\left((n+1)_{r}\right)^{1 / r}\right) t-(n+1) x\right] k_{n}(x, t),
$$


we have

$$
\begin{aligned}
x^{2}\left[T_{m}^{\widetilde{P}_{n, r}}(x)\right]^{\prime}= & \int_{0}^{\infty} x^{2}\left[k_{n}(x, t)\right]_{x}^{\prime}(t-x)^{m} d t-m x^{2} T_{m-1}^{\widetilde{P}_{n, r}}(x) \\
= & \int_{0}^{\infty}\left[\left((n+1)_{r}\right)^{1 / r} t-(n+1) x\right] k_{n}(x, t)(t-x)^{m} d t-m x^{2} T_{m-1}^{\widetilde{P}_{n, r}}(x) \\
= & \left((n+1)_{r}\right)^{1 / r} T_{m+1}^{\widetilde{P}_{n, r}}(x)+\left[\left((n+1)_{r}\right)^{1 / r}-(n+1)\right] x T_{m}^{\widetilde{P}_{n, r}}(x) \\
& -m x^{2} T_{m-1}^{\widetilde{P}_{n, r}}(x) .
\end{aligned}
$$

This completes the proof of recurrence relation. From recurrence relation by induction on $m$, it is easy to verify that the magnitude of the central moments satisfy $T_{m}^{\widetilde{P}_{n, r}}(x)=$ $O\left(n^{-[(m+1) / 2]}\right)$ for any $r \in \mathbb{N}$. The other consequences follow from the recurrence relation.

We have the following observations for our modified operator, corresponding to the estimate (1.3).

Let us suppose that the operators preserve the test functions $e_{1}, e_{2}, e_{3}, e_{4}$ respectively, then, by Lemma 2.2 for every continuous and bounded function $f$ on $(0, \infty)$, we have the following estimates:

$$
\begin{aligned}
& \left|\widetilde{P}_{n, 1}(f, x)-f(x)\right| \leq 2 \omega\left(f, \frac{x}{\sqrt{n+1}}\right), \\
& \left|\widetilde{P}_{n, 2}(f, x)-f(x)\right| \leq 2 \omega\left(f, \sqrt{2} \sqrt{\left.\left(1-\sqrt{\frac{n+1}{n+2}}\right) x\right)}\right. \\
& \left|\widetilde{P}_{n, 3}(f, x)-f(x)\right| \\
& \leq 2 \omega\left(f, \sqrt{\left(\frac{[(n+1)(n+2)]^{1 / 3}}{(n+3)^{2 / 3}}-2 \frac{(n+1)^{2 / 3}}{[(n+2)(n+3)]^{1 / 3}}+1\right) x}\right), \\
& \quad\left|\widetilde{P}_{n, 4}(f, x)-f(x)\right| \\
& \leq 2 \omega\left(f, \sqrt{\left(\frac{[(n+1)(n+2)]^{1 / 2}}{[(n+3)(n+4)]^{1 / 2}}-2 \frac{(n+1)^{3 / 4}}{[(n+2)(n+3)(n+4)]^{1 / 4}}+1\right) x}\right) .
\end{aligned}
$$

If we compare the above results, with the estimate (1.3), we find that the error becomes smaller and monotonically decreasing for $n \in \mathbb{N}, x \in(0, \infty)$, till the preservation of the test function $e_{3}$ as the following is true for second order moments:

$$
\begin{aligned}
\frac{\sqrt{n+2}}{n} & \geq \frac{1}{\sqrt{n+1}} \geq \sqrt{2} \sqrt{\left(1-\sqrt{\frac{n+1}{n+2}}\right)} \\
& >\sqrt{\left(\frac{[(n+1)(n+2)]^{1 / 3}}{(n+3)^{2 / 3}}-2 \frac{(n+1)^{2 / 3}}{[(n+2)(n+3)]^{1 / 3}}+1\right)},
\end{aligned}
$$


TABLE 1. Table for approximation

\begin{tabular}{|c|c|c|c|c|}
\hline $\mathrm{n}$ & $\sqrt{T_{2}^{\widetilde{P}_{n, 1}}(x)}$ & $\sqrt{T_{2}^{\widetilde{P}_{n, 2}}(x)}$ & $\sqrt{T_{2}^{\widetilde{P}_{n, 3}}(x)}$ & $\sqrt{T_{2}^{\widetilde{P}_{n, 4}}(x)}$ \\
\hline 5 & $0.408248 \mathrm{x}$ & $0.385175 \mathrm{x}$ & $0.378018 \mathrm{x}$ & $0.381509 \mathrm{x}$ \\
\hline 15 & $0.250000 \mathrm{x}$ & $0.244367 \mathrm{x}$ & $0.242538 \mathrm{x}$ & $0.243903 \mathrm{x}$ \\
\hline 25 & $0.196116 \mathrm{x}$ & $0.193356 \mathrm{x}$ & $0.192451 \mathrm{x}$ & $0.193203 \mathrm{x}$ \\
\hline 35 & $0.166667 \mathrm{x}$ & $0.164961 \mathrm{x}$ & $0.164399 \mathrm{x}$ & $0.164890 \mathrm{x}$ \\
\hline 40 & $0.156174 \mathrm{x}$ & $0.154767 \mathrm{x}$ & $0.154303 \mathrm{x}$ & $0.154716 \mathrm{x}$ \\
\hline 75 & $0.114708 \mathrm{x}$ & $0.114147 \mathrm{x}$ & $0.113961 \mathrm{x}$ & $0.114135 \mathrm{x}$ \\
\hline 10000 & $0.0099995 \mathrm{x}$ & $0.00999913 \mathrm{x}$ & $0.0099990 \mathrm{x}$ & $0.00999913 \mathrm{x}$ \\
\hline
\end{tabular}

but for higher order preservation of test function for example preservation of test function $e_{4}$, one can not have better approximation, which is also shown in the above table. Although the convergence takes places in all the cases for $n$ sufficiently large.

We prove below the direct estimate for the modified operators which preserve $e_{r}$. Let $\pi_{r}$ denote the space of all algebraic polynomials of degree $r$ and suppose $C_{B}(0, \infty)$ is the space of all continuous and bounded functions on $(0, \infty)$ endowed with the norm $\|f\|=\sup \{|f(x)|: x \in(0, \infty)\}$. Further let us consider the following $K$-functional:

$$
K_{2}(f, \delta)=\inf _{g \in C_{B}^{2}(0, \infty)}\left\{\|f-g\|+\delta\left\|g^{\prime \prime}\right\|\right\}
$$

where $\delta>0$ and $C_{B}^{2}(0, \infty)=\left\{g \in C_{B}(0, \infty): g^{\prime}, g^{\prime \prime} \in C_{B}(0, \infty)\right\}$.

Theorem 2.1. Let $f \in C_{B}(0, \infty)$, then for $r \in \mathbb{N}$ we have

$$
\left|\widetilde{P}_{n, r}(f, x)-f(x)\right| \leq C \omega_{2}\left(f, \sqrt{\delta_{n, r}}\right)+\omega\left(f,\left|\frac{(n+1)}{\left((n+1)_{r}\right)^{1 / r}}-1\right| x\right),
$$

where $C$ is a positive constant and $\delta_{n, r}$ is given as

$$
\delta_{n, r}=\left[\frac{(n+1)(2 n+3)}{\left((n+1)_{r}\right)^{2 / r}}-\frac{4(n+1)}{\left((n+1)_{r}\right)^{1 / r}}+2\right] x^{2} .
$$

Proof. We introduce the auxiliary operators $P_{n, r}: C_{B}(0, \infty) \rightarrow C_{B}(0, \infty)$ as follows

$$
P_{n, r}(f, x)=\widetilde{P}_{n, r}(f, x)-f\left(\frac{(n+1) x}{\left((n+1)_{r}\right)^{1 / r}}\right)+f(x) .
$$

These are linear operators and preserve linear functions. As by Lemma 2.1 and the positivity of $\widetilde{P}_{n, r}(t, x)$, we have

$$
P_{n, r}(t, x)=\widetilde{P}_{n, r}(t, x)-\frac{(n+1) x}{\left((n+1)_{r}\right)^{1 / r}}+x=\frac{(n+1) x}{\left((n+1)_{r}\right)^{1 / r}}-\frac{(n+1) x}{\left((n+1)_{r}\right)^{1 / r}}+x=x .
$$

Let $g \in C_{B}^{2}(0, \infty)$ and $x, t \in(0, \infty)$. By Taylor's formula, we have

$$
g(t)=g(x)+(t-x) g^{\prime}(x)+\int_{x}^{t}(t-u) g^{\prime \prime}(u) d u
$$


Then using Lemma 2.2 and by positivity of $\widetilde{P}_{n, r}$, we have

$$
\begin{aligned}
\left|P_{n, r}(g, x)-g(x)\right|= & \left|P_{n, r}\left(\int_{x}^{t}(t-u) g^{\prime \prime}(u) d u, x\right)\right| \\
= & \mid \widetilde{P}_{n, r}\left(\int_{x}^{t}(t-u) g^{\prime \prime}(u) d u, x\right) \\
& -\int_{x}^{\frac{(n+1) x}{((n+1) r)^{1 / r}}}\left(\frac{(n+1) x}{\left((n+1)_{r}\right)^{1 / r}}-u\right) g^{\prime \prime}(u) d u \mid \\
\leq & {\left[T_{2}^{\widetilde{P}_{n, r}}(x)+\left(\frac{(n+1) x}{\left((n+1)_{r}\right)^{1 / r}}-x\right)^{2}\right]\left\|g^{\prime \prime}\right\| } \\
:= & \delta_{n, r}|| g^{\prime \prime} \| .
\end{aligned}
$$

Next, by (2.7) and from Lemma 2.1, we have

$$
\left|P_{n, r}(f, x)\right| \leq \widetilde{P}_{n, r}(1, x)\|f\|+2\|f\| \leq 3\|f\| .
$$

Using (2.7), (2.8) and (2.9), we have

$$
\begin{aligned}
\left|\widetilde{P}_{n, r}(f, x)-f(x)\right| \leq & \left|P_{n, r}(f-g, x)-(f-g)(x)\right|+\left|P_{n, r}(g, x)-g(x)\right| \\
& +\left|f\left(\frac{(n+1) x}{\left((n+1)_{r}\right)^{1 / r}}\right)-f(x)\right| \\
\leq & 4|| f-g\left\|+\delta_{n, r}\right\| g^{\prime \prime}||+\left|f\left(\frac{(n+1) x}{\left((n+1)_{r}\right)^{1 / r}}\right)-f(x)\right| \\
\leq & C\left\{\|f-g\|+\delta_{n, r}|| g^{\prime \prime}||\right\}+\omega\left(f,\left|\frac{(n+1) x}{\left((n+1)_{r}\right)^{1 / r}}-x\right|\right) .
\end{aligned}
$$

Finally, if we take the infimum over all $g \in C_{B}^{2}(0, \infty)$, and using the inequality due to Gonska [8], $K_{2}(f, \delta) \leq C \omega_{2}(f, \sqrt{\delta}), \delta>0$, we get at once the desired result.

Corollary 2.1. Let $f \in \pi_{1}+C_{B}(0, \infty)$. Then

$$
\left|\widetilde{P}_{n, 1}(f, x)-f(x)\right| \leq C \omega_{2}\left(f, \frac{x}{\sqrt{(n+1)}}\right),
$$

where $C$ is certain positive constant.

Let us consider

$$
B_{2}(0, \infty):=\left\{f: f \in C(0, \infty) \rightarrow \mathbb{R} \text { and }|f(x)| \leq C(f)\left(1+x^{2}\right)\right\},
$$

where $C(f)$ is a positive constant depending only on $f$ and we denote $C_{2}(0, \infty)=$ $C(0, \infty) \cap B_{2}(0, \infty)$, by $C_{2}^{*}(0, \infty)$, we denote the subspace of all $f \in C_{2}(0, \infty)$ for which $\lim _{x \rightarrow \infty} \frac{f(x)}{1+x^{2}}<\infty$. The weighted modulus of continuity $\Omega(f, \delta)$ defined on infinite 
interval $\mathbb{R}^{+}$(see [11]) is defined as

$$
\Omega(f, \delta)=\sup _{|h|<\delta, x \in \mathbb{R}^{+}} \frac{|f(x+h)-f(x)|}{\left(1+h^{2}+x^{2}+h^{2} x^{2}\right)}, \quad \text { for each } f \in C_{2}(0, \infty) .
$$

We now estimate the following quantitative Voronovskaja-type asymptotic formula.

Theorem 2.2. Let $f^{\prime \prime} \in C_{2}^{*}(0, \infty)$ and $r \in \mathbb{N}$. Then for $x>0$, we have

$$
\begin{aligned}
& \mid \widetilde{P}_{n, r}(f, x)-f(x)-\left[\frac{(n+1)}{\left((n+1)_{r}\right)^{1 / r}}-1\right] x f^{\prime}(x) \\
& \quad-\left[\frac{(n+1)_{2}}{\left((n+1)_{r}\right)^{2 / r}}-2 \frac{(n+1)}{\left((n+1)_{r}\right)^{1 / r}}+1\right] \frac{x^{2}}{2} f^{\prime \prime}(x) \mid \\
& \leq C\left(1+x^{2}\right) \Omega\left(f^{\prime \prime}, n^{-1 / 2}\right)\left[T_{2}^{\widetilde{P}_{n, r}}(x)+n^{2} T_{6}^{\widetilde{P}_{n, r}}(x)\right],
\end{aligned}
$$

where $C$ is certain absolute constant.

Proof. By Taylor's expansion,

$$
f(t)=\sum_{i=0}^{2}(t-x)^{i} \frac{f^{(i)}(x)}{i !}+h(t, x)(t-x)^{2}
$$

where

$$
h(t, x):=\frac{f^{\prime \prime}(\eta)-f^{\prime \prime}(x)}{2}
$$

we have

$$
\begin{aligned}
\widetilde{P}_{n, r}(f, x)-f(x) & =\widetilde{P}_{n, r}((f(t)-f(x), x) \\
& =T_{1}^{\widetilde{P}_{n, r}}(x) f^{\prime}(x)+\frac{T_{2}^{\widetilde{P}_{n, r}}(x)}{2} f^{\prime \prime}(x)+\widetilde{P}_{n, r}\left(h(t, x)(t-x)^{2}, x\right),
\end{aligned}
$$

and $h$ is a continuous function which vanishes at 0 and $\eta$ lies between $x$ and $t$.

Proceeding along the lines of $[1$, Theorem 2], we have

$$
\widetilde{P}_{n, r}\left(|h(t, x)|(t-x)^{2}, x\right) \leq C\left(1+x^{2}\right) \Omega\left(f^{\prime \prime}, \delta\right)\left(T_{2}^{\widetilde{P}_{n, r}}(x)+\frac{1}{\delta^{4}} T_{6}^{\widetilde{P}_{n, r}}(x)\right) .
$$

Finally, using Lemma 2.2 and choosing $\delta=\frac{1}{\sqrt{n}}$, we get the desired result.

Corollary 2.2. Let $f^{\prime \prime} \in C_{2}^{*}(0, \infty)$, then for $x>0$, we have

$$
\begin{aligned}
& \left|\widetilde{P}_{n, 1}(f, x)-f(x)-\frac{x^{2}}{2(n+1)} f^{\prime \prime}(x)\right| \\
\leq & C\left(1+x^{2}\right)\left(\frac{x^{2}}{(n+1)}+\frac{n^{2} x^{6}}{(n+1)^{5}}\left(5 n^{2}+160 n+265\right)\right) \Omega\left(f^{\prime \prime}, n^{-1 / 2}\right),
\end{aligned}
$$

where $C$ is certain absolute constant. 


\section{Estimates for Polynomially Bounded Functions}

In this section, we are going to extend our point wise estimates for unbounded functions defined on $(0, \infty)$ and having polynomial growth of order greater than 2 . In recent years several authors in $[2,4,17]$ and [14] have studied such problems. We recall that in Section 4 , we used $\Omega(f ; \delta)$ appropriate for functions with polynomial growth of order at most 2. To overcome this R. Păltănea introduced in [14] the weighted modulus $\omega_{\varphi}(f ; h)$ defined as

$$
\omega_{\varphi}(f ; h)=\sup \left\{|f(x)-f(y)|: x \geq 0, y \geq 0,|x-y| \leq h \varphi\left(\frac{x+y}{2}\right)\right\}, \quad h \geq 0,
$$

where $\varphi(x)=\frac{\sqrt{x}}{1+x^{m}}, x \in(0, \infty), m \in \mathbb{N}, m \geq 2$. We consider here those functions, for which we have the property

$$
\lim _{h \rightarrow 0} \omega_{\varphi}(f ; h)=0 .
$$

It is easy to verify that this property is fulfilled for $f$ an algebraic polynomial of degree less than or equal to $m$. This follows from Theorem 2 in [14], which states that $\lim _{h \rightarrow 0} \omega_{\varphi}(f ; h)=0$ whenever the function $f \circ e_{2}$ is uniformly continuous on $(0,1]$ and the function $f \circ e_{v}, v=\frac{2}{2 m+1}$ is uniformly continuous on $[1, \infty)$, where $e_{v}(x)=x^{v}$, $x \geq 0$. Let us denote by $W_{\varphi}(0, \infty)$ the subspace of all real functions defined on $(0, \infty)$, for which the two conditions mentioned above hold true. In [17] we studied positive linear operators $L_{n}: E \rightarrow C(0, \infty)$, where $E$ is a subspace of $C(0, \infty)$, such that $C_{k}(0, \infty) \subset E$ with $k=\max \{m+3,6,2 m\}$ and

$C_{k}(0, \infty)=\left\{f \in C(0, \infty)\right.$ exists $M>0:|f(x)| \leq M\left(1+x^{k}\right)$ for all $\left.x>0\right\}, \quad k \in \mathbb{N}$.

One of main results of [17] which we are going to apply for $\widetilde{P}_{n, r}$ is the following quantitative estimate in terms of weighted modulus $\omega_{\varphi}(f ; h)$ (see [17, Theorem 2.2]).

Theorem 3.1 (Theorem A). Let $L_{n}: E \rightarrow C(0, \infty), C_{k}(0, \infty) \subset E, k=\max \{m+$ $3,6,2 m\}$ be sequence of linear positive operators, preserving the linear functions. If $f \in C^{2}(0, \infty) \cap E$ and $f^{\prime \prime} \in W_{\varphi}(0, \infty)$, then we have for $x \in(0, \infty)$ that

$$
\begin{aligned}
& \left|L_{n}(f, x)-f(x)-\frac{1}{2} f^{\prime \prime}(x) \mu_{n, 2}^{L}(x)\right| \\
\leq & \frac{1}{2}\left[\mu_{n, 2}^{L}(x)+\sqrt{2} \sqrt{L_{n}\left(\left[1+\left(x+\frac{|t-x|}{2}\right)^{m}\right]^{2} ; x\right)}\right] \omega_{\varphi}\left(f^{\prime \prime} ;\left(\frac{\mu_{n, 6}^{L}}{x}\right)^{1 / 2}\right) .
\end{aligned}
$$

Here $\mu_{n, k}^{L}(x)=L_{n}\left((t-x)^{k}, x\right)$ is the $k$-th order central moment of $L_{n}$.

We point out here that the statement in Theorem A can be extended for positive linear operators which don't preserve linear functions what we need in this case is to add the term $\mu_{n, 1}^{L}(x) f^{\prime}(x)$ in the left side of (3.1). As an application of Theorem A, we have the following result for modified Post-Widder operators. 
Theorem 3.2. Let $\widetilde{P}_{n, r}: E \rightarrow C(0, \infty), C_{k}(0, \infty) \subset E, k=\max \{m+3,6,2 m\}$, be the sequence of linear positive operators, preserving the test function $e_{r}$. If $f \in$ $C^{2}(0, \infty) \cap E$ and $f^{\prime \prime} \in W_{\varphi}(0, \infty)$, then we have for $x \in(0, \infty)$ that

$$
\begin{aligned}
& \mid \widetilde{P}_{n, r}(f, x)-f(x)-\left[\frac{(n+1)}{\left((n+1)_{r}\right)^{1 / r}}-1\right] x f^{\prime}(x) \\
& \quad-\frac{x^{2}}{2} f^{\prime \prime}(x)\left[\frac{(n+1)_{2}}{\left((n+1)_{r}\right)^{2 / r}}-2 \frac{(n+1)}{\left((n+1)_{r}\right)^{1 / r}}+1\right] \mid \\
& \leq \frac{1}{2}\left[\left(\frac{(n+1)_{2}}{\left((n+1)_{r}\right)^{2 / r}}-2 \frac{(n+1)}{\left((n+1)_{r}\right)^{1 / r}}+1\right) x^{2}\right. \\
& \left.\quad+\sqrt{2} \sqrt[\widetilde{P}_{n, r}\left(\left[1+\left(x+\frac{|t-x|}{2}\right)^{m}\right]^{2} ; x\right)]{ }\right] \\
& \quad \times \omega_{\varphi}\left(f^{\prime \prime} ;\left(\left[\frac{(n+1)_{6}}{\left((n+1)_{r}\right)^{6 / r}}-6 \frac{(n+1)_{5}}{\left((n+1)_{r}\right)^{5 / r}}+15 \frac{(n+1)_{4}}{\left((n+1)_{r}\right)^{4 / r}}\right.\right.\right. \\
& \left.\left.\left.\quad-20 \frac{(n+1)_{3}}{\left((n+1)_{r}\right)^{3 / r}}+15 \frac{(n+1)_{2}}{\left((n+1)_{r}\right)^{2 / r}}-6 \frac{(n+1)}{\left((n+1)_{r}\right)^{1 / r}}+1\right] x^{5}\right)^{1 / 2}\right) .
\end{aligned}
$$

We observe that the argument of $\omega_{\varphi}\left(f^{\prime \prime} ; \delta\right)$ in above theorem is of order $\delta=O\left(n^{-3 / 2}\right)$, $n \rightarrow \infty$.

Corollary 3.1. Under the assumption of above theorem, if the operators preserve test function $e_{1}$, then we have

$$
\begin{aligned}
& \left|\widetilde{P}_{n, 1}(f, x)-f(x)-\frac{x^{2}}{2(n+1)} f^{\prime \prime}(x)\right| \\
& \leq \frac{1}{2}\left[\frac{x^{2}}{n+1}+\sqrt{2} \sqrt{\widetilde{P}_{n, 1}\left(\left[1+\left(x+\frac{|t-x|}{2}\right)^{m}\right]^{2} ; x\right)}\right] \\
& \times \omega_{\varphi}\left(f^{\prime \prime} ;\left(\left[\frac{(n+2)_{5}}{(n+1)^{5}}-6 \frac{(n+2)_{4}}{(n+1)^{4}}+15 \frac{(n+2)_{3}}{(n+1)^{3}}\right.\right.\right. \\
& \left.\left.\left.-20 \frac{(n+2)_{2}}{(n+1)^{2}}+15 \frac{(n+2)}{(n+1)}-5\right] x^{5}\right)^{1 / 2}\right) \text {. }
\end{aligned}
$$

We consider $M_{n, k}^{L}=L_{n}\left(|t-x|^{k}, x\right)$ as the $k$-th order absolute moments of operators $L_{n}$.

The next main result of [17], which we are going to apply for the operators $P_{n, r}$ is the following quantitative variant of Voronovskaja theorem (see [17, Theorem 2.3]).

Theorem 3.3 (Theorem B). Let $L_{n}: E \rightarrow C(0, \infty), C_{k}(0, \infty) \subset E, k=\max \{m+$ $3,4\}$, be sequence of linear positive operators, preserving the linear functions. If 
$f \in C^{2}(0, \infty) \cap E$ and $f^{\prime \prime} \in W_{\varphi}(0, \infty)$, then we have for $x \in(0, \infty)$ that

$$
\begin{aligned}
& \left|L_{n}(f, x)-f(x)-\frac{1}{2} f^{\prime \prime}(x) \mu_{n, 2}^{L}(x)\right| \\
\leq & \frac{1}{2}\left[\mu_{n, 2}^{L}(x)+\frac{\sqrt{2}}{\sqrt{x}} \mu_{n, 2}^{L}(x) C_{n, 2, m}(x)\right] \omega_{\varphi}\left(f^{\prime \prime} ; \sqrt{\frac{\mu_{n, 4}^{L}(x)}{\mu_{n, 2}^{L}(x)}}\right),
\end{aligned}
$$

where

$$
C_{n, 2, m}(x)=1+\frac{1}{M_{n, 3}^{L}(x)} \sum_{k=0}^{m}\left(\begin{array}{c}
m \\
k
\end{array}\right) x^{m-k} \frac{M_{n, k+3}^{L}(x)}{2^{k}} .
$$

We suppose for the operators $L_{n}$ that

$$
\frac{M_{n, k}^{L}}{M_{n, 3}^{L}}, \quad 4 \leq k \leq m,
$$

is a bounded ratio for fixed $x$ and $m$, when $n \rightarrow \infty$.

Using Cauchy-Schwarz inequality, we have

$$
\widetilde{P}_{n, r}\left(|t-x|^{k}, x\right) \leq \sqrt{\widetilde{P}_{n, r}\left((t-x)^{2 k}, x\right)}
$$

Applying Lemma 2.2, we have

$$
\widetilde{P}_{n, r}\left(|t-x|^{k}, x\right)=O\left(n^{-k / 2}\right), \quad n \rightarrow \infty,
$$

and so it is easy to observe that $C_{n, 2, m}(x)$ is a bounded term for fixed $x$ and $m$ when $n \rightarrow \infty$. Also as in Theorem A, we point out that the statement in Theorem B can be extended in a similar way for positive linear operators, which don't preserve linear functions. As an application of Theorem B we obtain the following quantitative asymptotic Voronovskaja theorem for $\widetilde{P}_{n, r}$.

Theorem 3.4. Let $\widetilde{P}_{n, r}: E \rightarrow C(0, \infty), C_{k}(0, \infty) \subset E, k=\max \{m+3,4\}$, be sequence of linear positive operators, preserving the test function $e_{r}$. If $f \in C^{2}(0, \infty) \cap$ $E$ and $f^{\prime \prime} \in W_{\varphi}(0, \infty)$, then we have for $x \in(0, \infty)$ that

$$
\begin{aligned}
& \left|\widetilde{P}_{n, r}(f, x)-f(x)-T_{1}^{\widetilde{P}_{n, r}}(x) f^{\prime}(x)-\frac{T_{2}^{\widetilde{P}_{n, r}}(x)}{2} f^{\prime \prime}(x)\right| \\
\leq & \frac{T_{2}^{\widetilde{P}_{n, r}}(x)}{2}\left[1+\frac{\sqrt{2}}{\sqrt{x}} C_{n, 2, m}(x)\right] \omega_{\varphi}\left(f^{\prime \prime} ; x \sqrt{\frac{T_{4}^{\widetilde{P}_{n, r}}(x)}{T_{2}^{\widetilde{P}_{n, r}}(x)}}\right),
\end{aligned}
$$

where

$$
C_{n, 2, m}(x)=1+\frac{1}{M_{n, 3}^{\widetilde{P}_{n, r}}(x)} \sum_{k=0}^{m}\left(\begin{array}{c}
m \\
k
\end{array}\right) x^{m-k} \frac{M_{n, k+3}^{\widetilde{P}_{n, r}}(x)}{2^{k}} .
$$


Corollary 3.2. If $f, f^{\prime \prime}$ satisfy the same conditions as in the assumption of Theorem 3.4 , then we have for $x \in(0, \infty)$ that

$$
\lim _{n \rightarrow \infty} n\left[\widetilde{P}_{n, r}(f, x)-f(x)\right]=x f^{\prime}(x)+\frac{x^{2}}{2} f^{\prime \prime}(x) .
$$

Corollary 3.3. Under the assumption of above Theorem 3.4, if the operators preserve test function $e_{1}$, then we have

$$
\begin{aligned}
& \left|\widetilde{P}_{n, 1}(f, x)-f(x)-\frac{x^{2}}{2(n+1)} f^{\prime \prime}(x)\right| \\
\leq & \frac{x^{2}}{2(n+1)}\left[1+\frac{\sqrt{2}}{\sqrt{x}} C_{n, 2, m}(x)\right] \omega_{\varphi}\left(f^{\prime \prime} ; x \frac{\sqrt{3(n+3)}}{n+1}\right) .
\end{aligned}
$$

Our next goal in this section is to obtain estimates in terms of $K$-functional for polynomially bounded functions. We recall some notations from [4].

If $m \in \mathbb{N}$ is fixed we consider the weight

$$
\rho(x)=\rho_{m}(x)=(1+x)^{-m}, \quad x \in I \equiv[0, \infty) .
$$

The polynomials weighted space associated to $\rho$ is defined by

$$
C_{\rho}(I)=\left\{f \in C(I):\|f\|_{\rho}<\infty\right\},
$$

where

$$
\|f\|_{\rho}=\sup _{x \geq 0} \rho(x)|f(x)|
$$

In [4] it was used

$$
\phi(x)=\sqrt{(1+a x)(b x+c)}, \quad a \in \mathbb{N}_{0}, b>0, c \geq 0 .
$$

Here we set $a=b=c=1$, i.e.,

$$
\phi(x)=1+x .
$$

For $\lambda \in[0,1], s=1,2$, and $f \in C_{\rho}(I)$, we consider the $K$-functional

$$
K_{s, \phi^{\lambda}}(f, t)_{\rho}=\inf \left\{\|f-g\|_{\rho}+t^{s}\left\|\phi^{\lambda s} \cdot g^{(s)}\right\|_{\rho}, g \in W_{s, \lambda}^{\infty}(\phi)\right\},
$$

where $\left.W_{s, \lambda}^{\infty}(\phi)\right\}$ consists of all functions $g \in C_{\rho}(0, \infty)$ such that $g^{(s-1)}$ is absolutely continuous on every finite closed subinterval of $(0, \infty)$ and $\left\|\phi^{\lambda s} \cdot g^{(s)}\right\|_{\rho}<\infty$.

One of the main results in [4] is Theorem 1 which we cite here as (see [4, page 1498]).

Theorem 3.5 (Theorem C). For a positive integer $m, \rho(x)=(1+x)^{-m}$ and $\phi(x)=$ $\sqrt{(1+a x)(b x+c)}, a \in \mathbb{N}_{0}, b>0, c \geq 0$, and for positive linear operator $L_{n}: C_{\rho}(I) \rightarrow$ $C(I)$, we suppose the following conditions:

(i) $L_{n}\left(e_{0}\right)=e_{0}$;

(ii) there exist a constant $C_{1}$ and a sequence $\left\{\alpha_{n}\right\}, \alpha_{n} \rightarrow 0, n \rightarrow \infty$, such that

$$
L_{n}\left((t-x)^{2}, x\right) \leq C_{1} \alpha_{n} \phi^{2}(x) ;
$$


(iii) there exists a constant $C_{2}=C_{2}(m)$ such that for each $m \in \mathbb{N}$

$$
L_{n}\left((1+t)^{m}\right) \leq C_{2}(1+x)^{m}, \quad x \geq 0 ;
$$

(iv) there exists a constant $C_{3}=C_{3}(m)$, such that for each $m \in \mathbb{N}$

$$
\rho(x) L_{n}\left((t-x)^{2} / \rho(t) ; x\right) \leq C_{3} \alpha_{n} \phi^{2}(x), \quad x \geq 0 .
$$

Then for $\lambda \in[0,1]$ there exists a constant $C_{4}=C_{4}(m, \lambda)$ such that for any $f \in C_{\rho}(I)$, $x \in I, n \in \mathbb{N}$, one has

$$
\rho(x)\left|f(x)-L_{n}(f ; x)\right| \leq C_{4} K_{1, \phi^{\lambda}}\left(f ; \sqrt{\alpha_{n}} \phi^{1-\lambda}(x)\right)_{\rho}, \quad x \geq 0,
$$

where $K_{1, \phi^{\lambda}}(f ; t)_{\rho}$ is defined in (3.6) for $s=1$.

If in addition $L_{n}\left(e_{1}\right)=e_{1}$, then there exists a constant $C_{5}=C_{5}(m, \lambda)$ such that

$$
\rho(x)\left|f(x)-L_{n}(f ; x)\right| \leq C_{5} K_{2, \phi^{\lambda}}\left(f ; \sqrt{\alpha_{n}} \phi^{1-\lambda}(x)\right)_{\rho},
$$

where $K_{2, \phi^{\lambda}}(f ; t)_{\rho}$ is the $K$-functional defined in (3.6) for $s=2$.

We apply Theorem $\mathrm{C}$ for the modified Post-Widder operators $\widetilde{P}_{n, r}$. The condition (i) is trivial. The condition (ii) follows from the representation of second central moment $T_{2}^{\widetilde{P}_{n, r}}(x)$ in Lemma 2.2, with $\alpha_{n}=\frac{1}{n}$. The condition (iii) follows from representation of $\widetilde{P}_{n, r}\left(t^{k} ; x\right)=\frac{(n+1)_{k}}{\left((n+1)_{r}\right)^{k / r}} x^{k}$ obtained in previous section. To verify condition (iv) we apply Cauchy-Schwarz inequality and, by Lemma 2.2,

$$
\begin{aligned}
(1+x)^{-m} \widetilde{P}_{n, r}\left((t-x)^{2}(1+t)^{m} ; x\right) & \leq(1+x)^{-m} \sqrt{\widetilde{P}_{n, r}\left((t-x)^{4} ; x\right)} \sqrt{\widetilde{P}_{n, r}\left((1+t)^{2 m} ; x\right)} \\
& \leq(1+x)^{-m} C(r) T_{2}^{\widetilde{P}_{n, r}}(x) \sqrt{C_{2}(m)(1+x)^{2 m}} \\
& \leq C(r) \sqrt{C_{2}(m)} T_{2}^{\widetilde{P}_{n, r}}(x) \\
& \leq C_{3} \alpha_{n} \phi^{2}(x), \quad x \geq 0,
\end{aligned}
$$

where we have used condition (iii) and representation of $T_{2}^{\widetilde{P}_{n, r}}$ in Lemma 2.2. Therefore, as a consequence from Theorem $\mathrm{C}$, we obtain the following.

Theorem 3.6. Let $\rho(x)=(1+x)^{-m}, m \in \mathbb{N}, f \in C_{\rho}(I), \phi(x)=1+x, r \in \mathbb{N}, r \geq 2$, $\alpha=\frac{1}{n}$. Then for $\lambda \in[0,1]$ we have

$$
\rho(x)\left|\widetilde{P}_{n, r}(f ; x)-f(x)\right| \leq C(m, \lambda) K_{1, \phi^{\lambda}}\left(f ; \sqrt{\alpha_{n}} \phi^{1-\lambda}(x)\right)_{\rho} .
$$

For $r=1$ we have

$$
\rho(x)\left|\widetilde{P}_{n, 1}(f ; x)-f(x)\right| \leq C(m, \lambda) K_{2, \phi^{\lambda}}\left(f ; \sqrt{\alpha_{n}} \phi^{1-\lambda}(x)\right)_{\rho} .
$$

Remark 3.1. It is known (see [5]) that the $K$-functional $K_{s, \phi^{\lambda}}(f ; t)_{\rho}$ is equivalent to Ditzian-Totik moduli $\omega_{\phi^{\lambda}}^{s}(f ; t)_{\rho}$. In the most important cases $\lambda=0$ (point-wise Becker-type estimate) and $\lambda=1$ (estimate in norm) we can formulate (3.9) and (3.10) in terms of $\omega_{\phi^{\lambda}}^{s}(f ; t)_{\rho}$. If we denote by $C_{\rho, \infty}$ the set of all continuous functions 
on $(0, \infty)$ such that $\rho(x) f(x)$ has finite limit as $x \rightarrow \infty$, then it can be proved that $\lim _{t \rightarrow 0^{+}} \omega_{\phi^{\lambda}}^{s}(f ; t)_{\rho}=0$ whenever $f \in C_{\rho, \infty}(0, \infty)$. For $\lambda=0$ we get from here estimates (1.3) and (2.3)-(2.6) with some constant $C$ independent of $f$ and $n$ in place of 2 .

\section{Linear Combinations of Modified Post-Widder Operators}

From Corollary 2.1 and Corollary 3.2 we see that bounded continuous functions $f \in C_{B}(0, \infty)$ and also polynomially bounded functions can be approximated by $\widetilde{P}_{n, r}$ with order of approximation not greater than $O\left(n^{-1}\right)$. To increase the order of approximation we consider the linear combinations $L_{n, r}$ of $\widetilde{P}_{n, r}$. For more general settings and recent results for approximation by positive linear operators we refer the readers to the monograph [9]. Following the idea from [9] we will consider the following linear combinations

$$
L_{n, r}=\sum_{i=0}^{r} \alpha_{i}(n) \widetilde{P}_{n_{i}, r}
$$

where $n_{i}, i=0,1, \ldots$, are different positive numbers, $r \in \mathbb{N}$. Determine $\alpha_{i}(n)$ such that $L_{n, r} p=p$ for all $p \in \pi_{r}$ the set of algebraic polynomials of degree less than or equal to $r$. This seems to be natural as the operators $\widetilde{P}_{n, r}$ don't preserve linear functions if $r \geq 2$. The requirement that each polynomial of degree at most $r$ should be reproduced leads to the linear system

$$
L_{n, r}\left(t^{k}, x\right)=x^{k}, \quad 0 \leq k \leq r .
$$

From the nice representation of the images of monomials obtained in Section 2, $\widetilde{P}_{n, r}\left(t^{m}, x\right)=\frac{(n+1)_{m}}{\left((n+1)_{r}\right)^{m / r}} x^{m}$ and $(4.1)$, we obtain the system

$$
\left\{\begin{array}{l}
\alpha_{0}(n)+\alpha_{1}(n)+\alpha_{2}(n)+\cdots+\alpha_{r}(n)=1, \\
\sum_{i=0}^{r} \alpha_{i}(n) \frac{\left(n_{i}+1\right)_{m}}{\left(\left(n_{i}+1\right)_{r}\right)^{m / r}}=1, \quad 1 \leq m \leq r .
\end{array}\right.
$$

We observe that if $m=r$ in (4.3), the last equation coincides with the first one. So to have a unique solution for the coefficients $\alpha_{i}(n)$, we may impose additional condition

$$
L_{n, r}\left(t^{r+1}, x\right)=x^{r+1} .
$$

Then the system (4.3) will have the form

$$
\left\{\begin{array}{l}
\alpha_{0}(n)+\alpha_{1}(n)+\alpha_{2}(n)+\cdots+\alpha_{r}(n)=1, \\
\sum_{i=0}^{r} \alpha_{i}(n) \frac{\left(n_{i}+1\right)_{m}}{\left(\left(n_{i}+1\right)_{r}\right)^{m / r}}=1, \quad 1 \leq m \leq r-1, \\
\sum_{i=0}^{r} \alpha_{i}(n) \frac{\left(n_{i}+1\right)_{r+1}}{\left(\left(n_{i}+1\right)_{r}\right)^{(r+1) / r}}=1 .
\end{array}\right.
$$

Then from (4.5), we observe that all the polynomials of degree up to $r+1$ will be preserved by the combinations $L_{n, r}$ from (4.1). To obtain the direct estimate for 
approximation by linear combinations $L_{n, r}$, one need two additional conditions

$$
\begin{gathered}
n=n_{0}<n_{1}<n_{2}<\cdots<n_{r} \leq A n, \quad A=A(r), \\
\sum_{i=0}^{r}\left|\alpha_{i}(n)\right| \leq C .
\end{gathered}
$$

The condition (4.6) guarantees that

$$
L_{n, r}\left(|t-x|^{r+2}, x\right)=O\left(n^{-(r+2) / 2}\right), \quad n \rightarrow \infty
$$

The fact that all polynomials of degree less or equal to $r+1$ are preserved allow us to consider approximating functions $f$ from much larger class than $C_{B}(0, \infty)$, namely we consider $f \in \pi_{r+1}+C_{B}(0, \infty)$, where $\pi_{r+1}$ is the set of all algebraic polynomials of degree $\leq r+1$. Then following the same method applied by the authors in $[10$, Theorem 4] we arrive at the proof of following theorem.

Theorem 4.1. Let $f \in \pi_{r+1}+C_{B}(0, \infty)$. Then for every $x \in(0, \infty)$ and for $C>0$, $n>r$ and if the coefficients $\alpha_{i}(n)$ and numbers $n_{i}, 0 \leq i \leq r$, satisfy the conditions (4.5), (4.6) and (4.7), we have

$$
\left|L_{n, r}(f, x)-f(x)\right| \leq C \omega_{r+2}\left(f, n^{-1 / 2}\right) .
$$

Corollary 4.1. If $f \in \pi_{r+1}+C_{B}(0, \infty)$ and $f^{(r+2)} \in C_{B}(0, \infty)$, then

$$
\left|L_{n, r}(f, x)-f(x)\right| \leq C n^{-(r+2) / 2}|| f^{(r+2)} \|_{C_{B}(0, \infty)} .
$$

Let us consider the case $r=1$. In this case, to determine the coefficients $\alpha_{0}(n)$ and $\alpha_{1}(n)$ from (4.5), we get

$$
\left\{\begin{array}{l}
\alpha_{0}(n)+\alpha_{1}(n)=1, \\
\alpha_{0}(n) \frac{\left(n_{0}+1\right)_{2}}{\left(n_{0}+1\right)^{2}}+\alpha_{0}(n) \frac{\left(n_{1}+1\right)_{2}}{\left(n_{1}+1\right)^{2}}=1 .
\end{array}\right.
$$

The solution of this system is

$$
\alpha_{0}(n)=-\frac{n_{0}+1}{n_{1}-n_{0}}, \quad \alpha_{1}(n)=\frac{n_{1}+1}{n_{1}-n_{0}} .
$$

Obviously $\alpha_{1}(n)>0, \alpha_{0}(n)<0$. According to the conditions (4.6), (4.7), we must be careful with the choice of $\alpha_{0}(n), \alpha_{1}(n)$. For example, if $n_{0}=n, n_{1}=2 n$, then (4.11) implies

$$
\alpha_{0}(n)=-1-\frac{1}{n}, \quad \alpha_{1}(n)=2+\frac{1}{n},
$$

and (4.7) is satisfied. But if $n_{0}=n$ and $n_{1}=n+1$, then $\alpha_{0}(n)=-(n+1)$, $\alpha_{1}(n)=n+2$ and (4.7) is not fulfilled although (4.6) is true.

We observe that linear combinations $L_{n, 1}$ preserve $e_{0}, e_{1}, e_{2}$. Therefore, if $\pi_{2}$ denotes the space of all algebraic polynomials of degree 2, we may consider the approximating functions $f$ to be every $f \in \pi_{2}+C_{B}(0, \infty)$, which means that we consider $f=g+h$, where $g \in \pi_{2}$ is an arbitrary algebraic polynomial of degree less than or equal to 2 
and $g \in C_{B}(0, \infty)$ is an arbitrary bounded continuous function. With $\alpha_{0}, \alpha_{1}$ defined in (4.11) we have

Theorem 4.2. Let $f \in \pi_{2}+C_{B}(0, \infty)$. Then for every $C>0, n>1$, we have the following:

$$
\left|L_{n, 1}(f, x)-f(x)\right| \leq C \omega_{3}\left(f, n^{-1 / 2}\right) .
$$

If $f^{\prime \prime \prime} \in C_{B}(0, \infty)$, then

$$
\left|L_{n, 1}(f, x)-f(x)\right| \leq C n^{-3 / 2}|| f^{\prime \prime \prime}||_{C_{B}(0, \infty)} .
$$

Remark 4.1. We observe that the estimates with linear combinations $L_{n, r}$ from Theorem 4.1 and Corollary 4.1 are better than the previous estimates [16, (1.3)], also from [10]. The reason for this effect is that our modified Post-Widder operators $P_{n, r}$ preserve $e_{0}$ and $e_{r}$.

Acknowledgements. The authors are thankful to the reviewers for helpful remarks and suggestions which lead to essential improvement of the whole manuscript.

\section{REFERENCES}

[1] T. Acar, A. Aral and I. Rasa, The new forms of Voronovskaja's theorem in weighted spaces, Positivity 20 (2016), 25-40.

[2] A. Aral, H. Gonska, M. Heilmann and G. Tachev, Quantitative Voronovskaya-type results for polynomially bounded functions, Results Math. 70(3-4) (2016), 313-324.

[3] D. Aydin, A. Aral and G. B-Tunca, A generalization of Post-Widder operators based on qintegers, An. Stiint. Univ. Al. I. Cuza Iasi. Mat. (N.S.), DOI 10.2478/aicu-2014-0012.

[4] J. Bustamante, J. M. Quesada and L. M. de la Cruz, Direct estimate for positive linear operators in polynomial weighted spaces, J. Approx. Theory 162(8) (2010), 1495-1508.

[5] Z. Ditzian and V. Totik, Moduli of Smoothness, Springer-Verlag, New York, 1987.

[6] B. R. Draganov and K. G. Ivanov, A characterization of weighted approximation by the PostWidder and the Gamma operators, J. Approx. Theory 146 (2007), 3-27.

[7] B. R. Draganov and K. G. Ivanov, A characterization of weighted approximation by the Gamma and the Post-Widder operators, in: B. Bojanov, M. Drinov (Eds.), Proceedings of the International Conference on "Constructive Theory of Functions, Varna 2005", Academic Publishing House, Sofia, 2006, 80-87.

[8] H. Gonska, Quantitative Aussagen zur Approximation durch positive lineare Operatoren, $\mathrm{Ph} . \mathrm{D}$. Thesis, Universität Duisburg, Duisburg, 1979.

[9] V. Gupta and G. Tachev, Approximation with Positive Linear Operators and Linear Combinations, Series: Developments in Mathematics 50, Springer, Cham, 2017.

[10] V. Gupta and G. Tachev, Approximation by Szász-Mirakyan Baskakov operators, J. Appl. Funct. Anal. 9(3-4) (2014), 308-319.

[11] N. Ispir, On modified Baskakov operatos on weighted spaces, Turk. J. Math. 25 (2001), 355-365.

[12] S. Li and R. T. Wang, The characterization of the derivatives for linear combinations of Post Widder operators in $L_{p}$, J. Approx. Theory 97 (1999), 240-255.

[13] C. P. May, Saturation and inverse theorems for combinations of a class of exponential-type operators, Canad. J. Math. 28 (1976), 1224-1250.

[14] R. Pǎltănea, Estimates of approximation in terms of a weighted modulus of continuity, Bull. Transilv. Univ. Brasov 4(53) (2011), 67-74. 
[15] R. K. S. Rathore and O. P. Singh, On convergence of derivatives of Post-Widder operators, Indian J. Pure Appl. Math. 11 (1980), 547-561.

[16] L. Rempulska and M. Skorupka, On Approximation by Post-Widder and Stancu operators Preserving $x^{2}$, Kyungpook Math. J. 49 (2009), 57-65.

[17] G. Tachev and V. Gupta, General form of Voronovskaja's theorem in terms of weighted modulus of continuity, Results Math. 69(3-4) (2016), 419-430.

[18] D. V. Widder, The Laplace Transform, Princeton Mathematical Series, Princeton University Press, Princeton, New Jersey, 1941.

${ }^{1}$ Department of Mathematics,

Netaji Subhas University of Technology,

Sector 3 DWARKA, New Delhi 110078, India

Email address: vijaygupta2001@hotmail.com

${ }^{2}$ UNIVERSITY OF ARChiteCtURE,

Civil Engineering and Geodesy,

1 Hristo Smirnenski, Blvd., Sofia-1046, Bulgaria

Email address: gtt_fte@uacg.bg 